\title{
Remaining Useful Lifetime (RUL): Probabilistic Predictive Model
}

\author{
Ephraim Suhir \\ University of California, Santa Cruz, CA, 95064-1077, USA \\ suhire@aol.com
}

\begin{abstract}
Reliability evaluations and assurances cannot be delayed until the device (system) is fabricated and put into operation. Reliability of an electronic product should be conceived at the early stages of its design; implemented during manufacturing; evaluated (considering customer requirements and the existing specifications), by electrical, optical and mechanical measurements and testing; checked (screened) during fabrication; and, if necessary and appropriate, maintained in the field during the product's operation. Prognostics and health monitoring (PHM) effort can be of significant help, especially at the last, operational stage, of the product use. Accordingly, a simple and physically meaningful probabilistic predictive model is suggested for the evaluation of the remaining useful lifetime (RUL) of an electronic device (system) after an appreciable deviation from its normal operation conditions has been detected, and the corresponding increase in the failure rate and the change in the configuration of the wear-out portion of the bathtub curve has been assessed. The general concepts are illustrated by a numerical example. The model can be employed, along with other PHM forecasting and interfering tools and means, to evaluate and to maintain the high level of the reliability (probability of non-failure) of a device (system) at the operation stage of its lifetime.
\end{abstract}

\section{INTRODUCTION}

Reliability evaluation and assurance cannot be delayed until the device is fabricated and launched into operation, although it is sometime the case in many current practices. Reliability of an electronic product should be conceived at the early stages of its design; implemented during manufacturing; evaluated (considering customer requirements and the existing specifications), by electrical, optical and mechanical measurements and testing; checked (screened) during manufacturing (fabrication); and, if necessary and appropriate, maintained in the field during the product's operation (see. e.g., Suhir, 1997). The prognostics-and-healthmonitoring (PHM) concepts and techniques (see, e.g., Vichare and Pecht, 2006; Kirkland, Pombo, Nelson and
Berghout, 2004) are viewed today as an important part of electronic product reliability assurance at the last, operational, stage of the product's life, when there is a need and a possibility to maintain the product's high operational reliability in the field. As is known, PHM is based on a continuous monitoring of the products behavior in the field and is aimed at the prediction of the future reliability of the product from the detected and assessed deviation of its performance (because of aging, degradation, elevated loading condition, extraordinary and harsh environment, etc.) from the normal (specified) performance. The ability to predict the remaining useful lifetime (RUL), after a certain malfunction is detected or anticipated, is one of the most crucial PHM objectives.

Accordingly, in the analysis that follows, we suggest a formalism for the assessment of the RUL of a device (system) from 1) the given steady-state failure rate (FR), 2) agreed-upon ultimate failure rate (beyond which the further use of the device or a system is deemed undesirable), 3) the increase in the FR at the wear-out portion of the bathtub curve and 4) the detected or anticipated small "jump" in the FR that determines the beginning of the actual wear-out stage. This jump could be determined in many ways, depending on the physical nature of the addressed degradation (aging) process, typical loads (usually thermally induced), available PHM equipment and its trustworthiness, etc. Having in mind the inevitable uncertainties in the magnitude of such a "jump", we use, in addition to the "deterministic" formalism, also a formalism based on the probabilistic design-for-reliability (PDfR) concept (see Suhir, 2010 and Suhir, Mahajan, Lucero and Bechou, 2012).

\section{ANALYSIS}

\subsection{Remaining Useful Lifetime (RUL)}

Let the wear-out portion $\lambda(t)$ of the original (specified) bathtub curve is configured as shown in Fig.1 (solid line). The commencement of this portion is defined by the moment of time when a (typically insignificant) malfunction in the device performance is detected, and the PHM instrumentation and algorithms predict a small 
"jump" $\Delta \lambda$ in the bathtub curve. The new (corrected) configuration $\tilde{\lambda}(t)$ of the wear out portion of the bathtub curve is shown as a broken line in Fig.1. The "healthy" and "damaged" configurations of this portion of the bathtub curve can be approximated as

$$
\lambda(t)=\lambda_{0} t^{n}, \quad \tilde{\lambda}(t)=\tilde{\lambda}_{0} t^{\tilde{n}}
$$

respectively. The following notation is used:

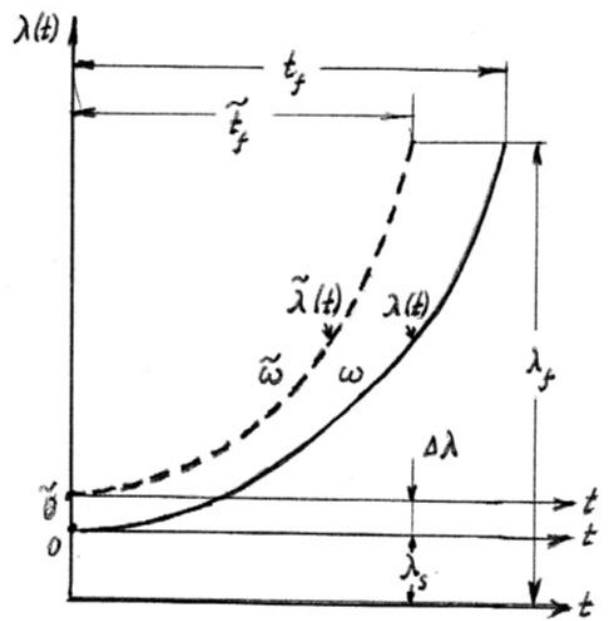

Fig.1. Specified/"healthy" (solid line) and the deviated/"damaged" (broken line) configurations of the wear out portion of the bathtub curve.

$$
\begin{gathered}
\lambda_{0}=\frac{\lambda_{f}-\lambda_{s}}{t_{f}^{n}}, \\
n=\frac{\lambda_{f}-\lambda_{s}}{\omega} t_{f}-1=\frac{1-\beta}{\beta}, \\
\tilde{\lambda}_{0}=\frac{\lambda_{f}-\lambda_{s}-\Delta \lambda}{\tilde{t}_{f}^{\tilde{n}}}, \\
\tilde{n}=\frac{\lambda_{f}-\lambda_{s}-\Delta \lambda}{\tilde{\omega}} \tilde{t}_{f}-1=\frac{1-\tilde{\beta}}{\tilde{\beta}},
\end{gathered}
$$

$\lambda_{f}$ is the specified maximum acceptable failure rate beyond which the further use of the device or a system is deemed unfeasible, $\lambda_{s}$ is the steady-state failure rate, $t_{f}$ is the specified duration of the wear-out period of the bath-tub curve, $\omega$ is the (shaded) area under the curve $\lambda(t), \quad \beta=\frac{1}{n+1}$ is the area coefficient (reflecting the degree of deviation of the area in question from a rectangular), $\tilde{t}_{f}$ is the RUL, $\tilde{\omega}$ is the (shaded) area under the curve $\tilde{\lambda}(t)$ and $\tilde{\beta}=\frac{1}{\tilde{n}+1}$ is the corresponding area coefficient. The first and the third formulas in (2) are, in effect, conditions that reflect the requirement that the device is not supposed to be operated beyond the $\lambda_{f}$ FR level.

In an approximate analysis, in order to assess the sensitivity of the $\tilde{t}_{f}$ value to the change in the major factors affecting the RUL after an appreciable deviation from the specified ("standard", "anticipated") wear out portion of the bathtub curve has been diagnosed and assessed, we assume $\lambda_{0} \approx \tilde{\lambda}_{0}$ and $\beta \approx \tilde{\beta}$. Then the first and the third formulas in (2) result in the following formula for the relative RUL:

$$
\tau_{u}=\frac{\tilde{t}_{f}}{t_{f}} \approx(1-\xi)^{\frac{\beta}{1-\beta}},
$$

where

$$
\xi=\frac{\Delta \lambda}{\lambda_{f}-\lambda_{s}}
$$

is the ratio of the predicted increase in the failure rate to the difference between the ultimate failure rate and the steady state failure rate.

The following conclusions can be made based on the formula (3):

- The relative RUL $\tau_{u}$ changes from one to zero, when the predicted damage ratio $\xi$ changes from zero to one.

- The relative RUL $\tau_{u}$ changes from $1-\xi$ to zero, when the "fullness" parameter $\beta$ changes from 0.5 to one. The condition $\frac{d \tau_{u}}{d \beta}=0$ indicates that the $\tau_{u}=1-\xi$ value is the maximum possible value of the $\tau_{u}$ ratio. This value takes place for zero $\xi$ values, i.e., for zero "damages" $\Delta \lambda$.

- The relative RUL increases with an increase in the parameter $\beta$ (decrease in the exponent $n$ ), i.e., with an increase in the "fullness" of the wear-out portion of the bathtub curve. In other words, it is advisable that the wear-out portion of the bathtub curve "concentrates" at the end of the bathtub diagram;

- The relative RUL increases with a decrease in the predicted "disturbance" $\Delta \lambda$ and the increase in the difference between the ultimate (specified) failure rate $\lambda_{f}$ and the steady-state failure rate $\lambda_{s}$. 
- Lower steady-state failure rates $\lambda_{s}$ result in larger RUL.

These intuitively more or less obvious conclusions are quantified by the formulas (3) and (4).

Let us show, as an illustration, how the possible (expected) "damage" $\Delta \lambda$, if attributed to the change in temperature, can be assessed from the detected appreciable increase $\Delta T$ in temperature. If one chooses the Boltzmann-Arrhenius law

$$
\bar{\tau}=\tau_{0} \exp \left(\frac{U}{k T}\right)
$$

to determine the mean-time-to-failure $\bar{\tau}$ and the corresponding failure rate $\lambda=\frac{1}{\bar{\tau}}$ for the given absolute temperature $T$, then the derivative $\frac{d \lambda}{d T}$ can be found as

$$
\frac{d \lambda}{d T}=-\frac{U}{\tau_{0} k T^{2}} \exp \left(-\frac{U}{k T}\right)
$$

Here $U$ is the activation energy, $k$ is Boltzmann's constant, $\lambda_{s}$ is the steady-state failure rate, and $T_{s}$ is the steady-state temperature (prior to the detected temperature increase). Replacing the differentials in the above formula with finite differences, we have:

$$
\Delta \lambda=-\frac{U}{\tau_{0} k T^{2}} \exp \left(-\frac{U}{k T}\right) \Delta T=-\frac{U \lambda_{s}}{k T_{s}^{2}} \Delta T \cdot(7)
$$

The formula (7) indicates particularly that the "damage" will be lower for lower steady-state failure rates and for higher steady-state operation temperatures.

\subsection{Probabilistic Approach}

The probabilistic design for reliability (PDfR) approach enables one to account for the random nature of the relative RUL $\tau_{u}$ as a non-random function of the random variable $\xi$.

Let, for the sake of simplicity, assume that this variable is distributed in accordance with the Rayleigh's law:

$$
f_{\xi}(\xi)=\frac{\xi}{\xi_{0}^{2}} \exp \left(-\frac{\xi^{2}}{2 \xi_{0}^{2}}\right),
$$

where $\xi_{0}$ is the most likely (deterministic) value of the variable $\xi$. The physical justification for the taken assumption that the random variable $\xi$ is distributed in accordance with the Rayleigh's law is that a random variable of time should always be positive, that low $\xi$ values are more likely than high $\xi$ values, that the zero value of the variable $\xi$ should be equal to zero, and the probabilities of its high values should be very small and should decrease with an increase in the $\xi$ value. Weibull distribution and normal distributions with significant mean-to-standard-deviation ratios could be assumed in practical applications as more flexible and "richer" twoparametric distributions for the variable $\xi$.

The probability density function of the variable $\tau_{u}$ can be found as

$$
\begin{aligned}
& f_{\tau}\left(\tau_{u}\right)= \\
& =\frac{1-\beta}{\beta \xi_{0}^{2}} \tau_{u}^{\frac{1}{\beta}-2}\left(1-\tau_{u}^{\frac{1}{\beta}-1}\right) \exp \left[-\frac{\left(1-\tau_{u}^{\frac{1}{\beta}-1}\right)^{2}}{2 \xi_{0}^{2}}\right],
\end{aligned}
$$

and the probability distribution function is

$$
F_{\tau}\left(\tau_{u}\right)=\exp \left[-\frac{\left(1-\tau_{u}^{\frac{1}{\beta}-1}\right)^{2}}{2 \xi_{0}^{2}}\right]
$$

This function determines the probability that the random RUL ratio (3) will not exceed a certain $\tau_{u}$ level. It is clear that one wishes to assure that this probability is as low as possible.

When the PDfR approach is used, there is of interest to compare the probabilities of non-failure of the "healthy" and the "damaged" device (system). Assuming, again, for the sake of simplicity, that the exponential law of reliability is applicable, one can use the following formula to evaluate the probability of non-failure:

$$
P=\exp (-\lambda t) \text {. }
$$

The time runs faster, by the factor of $1 / \tau$, in the case of a "damaged" device, so that the formula (11) yields:

$$
\widetilde{P}=P^{1 / \tau}
$$

where the $\tau$ ratio is defined by the formula (3).

\section{NUMERICAL EXAMPLE}

\section{Input data:}

Steady-state failure rate: $\lambda_{s}=2.5 \times 10^{-5} 1 / \mathrm{hr}$;

Ultimate failure rate: $\lambda_{u}=7.5 \times 10^{-5} 1 / \mathrm{hr}$;

Area ("fullness") parameter of the wear-out portion of the bathtub curve: $\beta=0.75$

\section{Calculated data:}

The calculated data for the relative RUL $\tau_{u}$ ratios for different changes $\Delta \lambda$ in the initial values are shown in Table 1. These data indicate that the RUL of the "damaged" device (system) rapidly decreases with an increase in the level of the initial "damage". This 
"damage" is defined in our analysis as the relative deviation of the failure rate at the wear-out portion of the bathtub curve.

\begin{tabular}{|l|c|c|c|c|c|c|}
\hline$\Delta \lambda$ & & & & & & \\
$l / h r$ & 0.10 & 0.25 & 0.75 & 1.0 & 1.5 & 2.5 \\
E-5 & E-5 & E-5 & E-5 & E-5 & E-5 \\
\hline$\xi$ & 0.02 & 0.05 & 0.15 & 0.20 & 0.30 & 0.5 \\
\hline$\tau_{u}$ & 0.9412 & 0.8574 & 0.6141 & 0.6672 & 0.3430 & 0.125 \\
\hline
\end{tabular}

Table 1. Calculated relative remaining useful life (RUL) data vs. change in the failure rate

\begin{tabular}{|c|c|c|c|c|c|}
\hline $\boldsymbol{\xi}_{0}$ & $\mathbf{0 . 0 2}$ & $\mathbf{0 . 0 4}$ & $\mathbf{0 . 0 6}$ & $\mathbf{0 . 0 8}$ & $\mathbf{0 . 1 0}$ \\
\hline$\tau_{u}$ & $\mathbf{x}$ & $\mathbf{x}$ & $\mathbf{x}$ & $\mathbf{x}$ & $\mathbf{x}$ \\
\hline $\mathbf{0 . 2}$ & 0 & 0 & 0 & 0 & 0.0002 \\
\hline $\mathbf{0 . 4}$ & 0 & 0 & 0 & 0.0045 & 0.0313 \\
\hline $\mathbf{0 . 6}$ & 0 & 0.0005 & 0.0332 & 0.1472 & 0.2935 \\
\hline $\mathbf{0 . 8}$ & 0.001625 & 0.2008 & 04899 & 0.6694 & 0.7734 \\
\hline $\mathbf{0 . 9}$ & 0.2257 & 0.6892 & 0.8475 & 0.9112 & 0.9422 \\
\hline $\mathbf{1 . 0}$ & 1.0 & 1.0 & 1.0 & 1.0 & 1.0 \\
\hline
\end{tabular}

Table 2. Calculated probabilities of the situation that the actual remaining-useful-life (RUL) ratio for the "damaged" and "healthy" devices is below the remaining relative useful life level

The calculated probabilities $F\left(\tau_{u}\right)$ of the situation that the actual ratio of the RUL of the "damaged" and "healthy" devices remains below the $\tau_{u}$ level is calculated for different $\tau_{u}$ values and different most likely values $\xi_{0}$ of the relative damage $\xi$ are shown in Table 2 . The calculated data indicate that the sought probability is small indeed (which is certainly a desirable situation) for low $\tau_{u}$ and low $\xi_{0}$ values, when the most likely damage $\xi_{0}$ is small and/or when the RUL of the damaged device is short compared to the RUL of the "healthy" device. This means that the probability $P\left(\tau_{u}\right)=1-F\left(\tau_{u}\right)$ that the actual RUL ratio will exceed the $\tau_{u}$ value indicated in the left column of the Table 2 is high.

\begin{tabular}{|c|c|c|c|c|c|c|}
\hline$\tau_{u}$ & 0.2 & 0.4 & 0.6 & 0.8 & 0.9 & 1.0 \\
\hline$P$ & $\mathbf{x}$ & $\mathbf{x}$ & $\mathbf{x}$ & $\mathbf{x}$ & $\mathbf{x}$ & $\mathbf{x}$ \\
\hline E-2 & E-10 & E-5 & $\begin{array}{l}4.642 \\
\text { E-4 }\end{array}$ & $\begin{array}{l}3.162 \\
\text { E-3 }\end{array}$ & $\begin{array}{l}5.995 \\
\text { E-3 }\end{array}$ & E-2 \\
\hline E-3 & E-15 & $\begin{array}{l}3.162 \\
\text { E- } 8\end{array}$ & E-5 & $\begin{array}{l}1.778 \\
\text { E-4 }\end{array}$ & $\begin{array}{l}4.642 \\
\text { E-4 }\end{array}$ & E-3 \\
\hline E-4 & E-20 & E-10 & $\begin{array}{l}2.154 \\
\text { E-7 }\end{array}$ & E-5 & $\begin{array}{l}3.594 \\
\text { E-5 }\end{array}$ & E-4 \\
\hline E-5 & E-25 & $\begin{array}{l}3.16 \\
\text { E-13 }\end{array}$ & $\begin{array}{l}4.642 \\
\text { E-9 }\end{array}$ & $\begin{array}{l}5.623 \\
\text { E-7 }\end{array}$ & $\begin{array}{l}2.783 \\
\text { E-6 }\end{array}$ & E-5 \\
\hline
\end{tabular}

Table 3. Calculated decrease in the probability $\tilde{P}_{\text {of non- }}$ failure, at the given level of the failure rate, of the "damaged" device as compared to the probability $P$ of non-failure of the "healthy" one.

As to the data at the lower right corner of the Table 3, they indicate that it is very likely that the actual RUL of the damaged device will be considerably lower than the desirably high $\tau_{u}$ value. The Table 3 , obtained on the basis of the formula (12), shows the decrease in the probability $\tilde{P}$ of non-failure, at the given level of the failure rate, of the "damaged" device as compared to the probability $P$ of non-failure of the "healthy" one. The probabilities $\tilde{Q}=1-\tilde{P}$ of failure are high for low $\tau_{u}$ ratios, especially if the specified probabilities $P$ of nonfailure for a "healthy" device are low.

\section{CONCLuSiON}

Simple, easy-to-use and physically meaningful predictive formalisms are developed for the evaluation of the remaining useful life (RUL) after an appreciable deviation from the normal operation conditions has been detected and the change in the wear-out portion of the bathtub curve has been predicted. Both deterministic and probabilistic approaches are considered. The models can be used, in addition to other PHM forecasting means, in the analysis and design of various PHM systems, including the PDfR.

\section{REFERENCES}

Kirkland, L.V., Pombo, T, Nelson, K., Berghout, F. (2004). Avionics Health Management: Searching for the Prognostics Grail, Proceedings of IEEE Aerospace Conference, Vol. 5. Big Sky, MO

Suhir, E. (1997). Applied Probability for Engineers and Scientists, McGraw-Hill

Suhir, E. (2010). Probabilistic Design for Reliability, ChipScale Reviews, vol.14, No.6.

Suhir, E., Mahajan, R., Lucero, A. and Bechou, L. (2012). Probabilistic Design-for-Reliability Concept and Novel Approach to Qualification Testing of Aerospace Electronic Products, Proceedings of IEEE Aerospace Conference, Vol. 5. Big Sky, MO (to be presented and published)

Vichare, N., Pecht, M. (2006). Prognostics and Health Management of Electronics, IEEE Transactions on Components and Packaging Technologies, Vol. 29, No. 1 


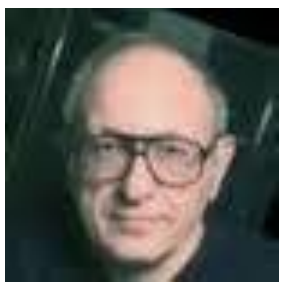

Ephraim Suhir is on the faculty of the University of California, Santa Cruz, CA, Electrical Engineering Department. $\mathrm{He}$ is also Visiting Professor, Mechanical Engineering Department, University of Maryland, College Park, MD and Department of Electronics Materials, Technical University, Vienna, Austria. He is Fellow of the Institute of Electrical and Electronics Engineers (IEEE), the American Physical Society (APS), the American Society of Mechanical Engineers (ASME), the Institute of Physics (IoP), UK, and the Society of Plastics Engineers (SPE). Dr. Suhir has been elected as Foreign Full Member (Academician) of the National Academy of Engineering and Technological Sciences, Ukraine. $\mathrm{He}$ is on the US Department of State roster as Fulbright Scholar in information and telecommunication technologies.

Dr. Suhir is a co-founder of the ASME Journal of Electronic Packaging and served as its Technical Editor (Editor-inChief) for eight years (1993-2001). He holds 22 US patents and has authored about 300 technical publications (papers, book chapters, books), including monographs "Probabilistic Methods in Ship Structural Analysis", Nikolayev Institute of Naval Architecture, Nikolayev, Ukraine, 1973 (in Russian), "Structural Analysis in Microelectronics and Fiber Optics", Van-Nostrand, 1991, and "Applied Probability for Engineers and Scientists", McGraw-Hill, 1997. Dr. Suhir is editor of the Springer book series on physics, mechanics and packaging of microelectronic and photonic systems. He is
Distinguished Lecturer of the IEEE CPMT (Components, Packaging and Manufacturing Technology) Society, serves on several Technical Committees of this Society and is Associate Editor of the IEEE CPMT Transactions on Advanced Packaging.

Dr. Suhir received many distinguished service and professional awards, including: 2004 ASME Worcester Read Warner Medal for outstanding contributions to the permanent literature of engineering through a series of papers in Mechanical, Microelectronic, and Optoelectronic Engineering, and is the third Russian American (after Igor Sikorsky and Stephen Timoshenko) who received this prestigious award; 2001 IMAPS John A. Wagnon Technical Achievement Award for outstanding contributions to the technical knowledge of the microelectronics, optoelectronics, and packaging industry; 2000 IEEE-CPMT Outstanding Sustained Technical Contribution Award for outstanding, sustained and continuing contributions to the technologies in fields encompassed by the CPMT Society; 2000 SPE International Engineering/Technology (Fred O. Conley) Award for outstanding pioneering and continuing contributions to plastics engineering; 1999 ASME and PiTau-Sigma Charles Russ Richards Memorial Award for outstanding contributions to mechanical engineering, and 1996 Bell Laboratories Distinguished Member of Technical Staff Award for developing engineering mechanics methods for predicting the reliability, performance, and mechanical behavior of complex structures used in manufacturing AT\&T and Lucent Technologies products. 